\title{
The sonoanatomical relation of trunk level of brachial plexus at supraclavicular fossa
}

Keisuke Matsuo ${ }^{1)}$, Tomoaki Yanaru(2), Yuta Suenaga ${ }^{3)}$, Ken Yamaura ${ }^{3)}$ 1) Kagoshima University Hospital, 2) Fukuoka-Higashi Medical Center, 3) Fukuoka University Hospital

\section{Background and Goal of Study}

Supraclavicular brachial plexus block is usually done trunk level of brachial plexus. Understanding of the relation of trunks is important for patient to receive good postoperative pain control.

However, there is no article investigated the anatomical relation of trunks.

The aim of this study was to investigate the relation of trunks at supraclavicular fossa by ultrasonography.

\section{Materials and Methods}

This study was approved by the Ethics Committee of Fukuoka University Hospital. (IRB number: 15-8-06)

One hundred patients scheduled for elective orthopedic surgery were participated in this study.

Patients were placed in the supine position, with the head facing away from the side to be observed.

The brachial plexus was observed from the level of C5 vertebra to the supraclavicular fossa by ultrasonography.

Distributions of trunks were defined as follow.
Superior trunk derives:
$\mathrm{C} 5$ and $\mathrm{C} 6$ roots
Middle trunk derives:
C7 root
$\mathrm{C} 8$ and $\mathrm{T} 1$ roots

Inferior trunk derives:

Location of trunks at supraclavicular fossa were defined as follow.

Group A: Superior, middle and inferior trunks are located from anterior to posterior (Fig. 1)

Group B: Superior and middle trunks are located side by side at anterior area and inferior trunk is located at inferior area (Fig. 2)

\section{Result}

The relation of trunks is summarized in table 1 .

The frequencies of Group A and Group B are $76.5 \%$ and $23.5 \%$.

Table 1. The frequencies of Group A and B (\%)

\begin{tabular}{cccc}
\hline & Right & Left & Total \\
\hline Group A & 74 & 79 & 76.5 \\
Group B & 26 & 21 & 23.5 \\
\hline
\end{tabular}

\section{Disucussion}

If the catheter tip of supraclavicular brachial plexus block was inserted below sternocleidomastoid muscle and above brachial plexus for shoulder surgery, it is effective for superior trunk and maintained good postoperative pain of Group A. However, if the catheter tip is inserted more inside near the subclavian artery, local anesthetics are only effective to middle trunk and it does not control pain management of Group B.

Inferior trunk is located at inferior area both Group A and B. The catheter tip is inserted below the subclavian artery, it is possible to control pain management for ulnar side of arm.

\section{Conclusion}

Understanding of the relation of trunks at supraclavicular fossa is important for patient to maintain good postoperative pain control.

\section{References}

A Double-Blind Randomized Comparison of Continuous Interscalene, Supraclavicular, and Suprascapular Blocks for

Reg Anesth Pain Med Auyong DB, et al.

Phrenic palsy and analgesic quality of continuous supraclavicular vs. interscalene plexus blocks after shoulder surgery. Acta Anaesthesiol Scand 2016; 60: 1142-51. Wiesmann T,et al.

A randomized comparison of infraclavicular and supraclavicular continuous peripheral nerve blocks for postoperative A

Reg Anesth Pain Med 2011; 36: 26-31. Mariano ER,et al.

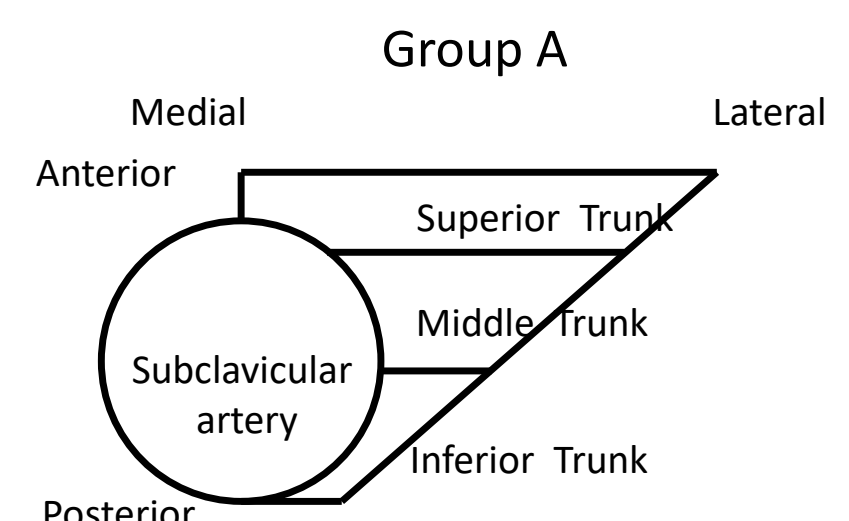

Fig.1 The relation of trunks at supraclavicular fossa.Group $A$ is that superior,middle and inferior trunk are located from anterior to posterior.
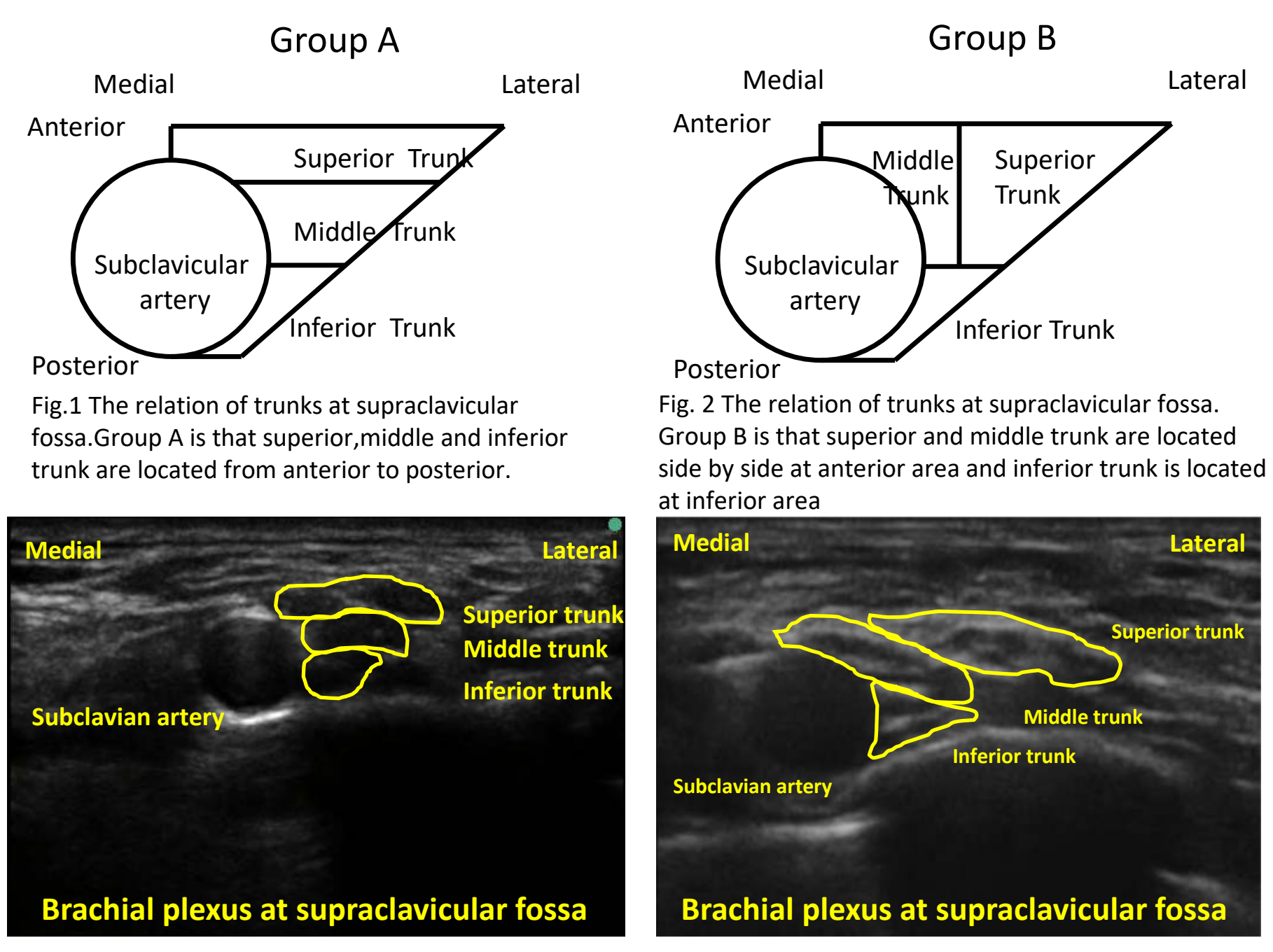